\title{
The Nainital Cape Survey Project : A Search for Pulsation in Chemically Peculiar Stars
}

\author{
Nand Kumar Chakradhari ${ }^{1 *}$, Santosh Joshi ${ }^{2 \dagger}$ \\ ${ }^{1}$ SoS in Physics and Astrophysics, Pt. R. S. University, Raipur, 492010, India \\ ${ }^{2}$ Aryabhatta Research Institute of Observational Sciences (ARIES), Nainital, 2631002, India
}

\begin{abstract}
The Nainital-Cape Survey is a dedicated search programme initiated in 1999 in the coordination of astronomers from SAAO South Africa, ARIES Nainital and ISRO Bangalore. Over the last 17 years a total of 345 chemically peculiar stars were monitored for photometric variability, making it one of the longest groundbased survey to search for pulsation in chemically peculiar stars in terms of both time span and sample size. Under this survey, we discovered rapid pulsation in the Ap star HD12098 while $\delta$ Scuti-type pulsations were detected in seven Am stars. Those stars in which pulsations were not detected have also been tabulated along with their detailed astrophysical parameters for further investigation.
\end{abstract}

\section{Introduction}

Stars having anomalous line strengths (strong/weak) in their optical spectra are called chemically peculiar (CP) stars. They are mostly A-type stars, but some of them are also found in spectral class from late B to early F type (Wolf 1983). These stars are grouped as Am/Fm (CP1), Ap/Bp (CP2), $\mathrm{Hg}-\mathrm{Mn}$ (CP3), and He weak/strong (CP4) stars (Preston 1974). Since both Am and Ap stars are of prime interest for the present study, we introduce them briefly here. The Am stars are relatively cool (6 500-10000 K) stars exhibiting weak lines of Ca, Sc and strong lines of Sr, Eu, and other rare-earth elements. Some stars of this group show pulsations similar to those observed in $\delta$ Scuti ( $\delta$ Sct) stars (Joshi et al. 2003, 2006, 2009; Smalley et al. 2011; Catanzaro \& Ripepi 2014; Hou et al. 2015). Similarly, the Ap stars have a surface overabundance of $\mathrm{Sr}, \mathrm{Cr}, \mathrm{Eu}$, and other rare earth elements, a high magnetic field (few $\mathrm{kG}$ ) and effective temperatures ranging from 7000 to $10000 \mathrm{~K}$. In the early 1980s, it was found that a subset of Ap stars exhibit short period pulsations in the range of 5 to 23 min and are termed as rapidly oscillating Ap (roAp) stars (Kurtz \& Martinez 2000). The roAp stars are important tools for asteroseismology.

The $\delta$ Sct stars show radial and/or non-radial low-overtone $p$-mode pulsations while roAp stars exhibit low-amplitude, low-degree, high-overtone, non-radial $p$-mode pulsations. The photometric amplitude of oscillations in roAp star is modulated with rotation and can be explained by the oblique pulsator model (Kurtz 1982). The multi-periodic nature of these pulsating Ap/Am stars make them suitable for asteroseismic studies (Joshi \& Joshi 2015). The Nainital-Cape Survey, initiated in 1999

*nkchakradhari@gmail.com

${ }^{\dagger}$ santosh@aries.res.in 
and coordinated by astronomers from the South African Astronomical Observatory (SAAO, Sutherland, South Africa), the Aryabhatta Research Institute of Observational Sciences (ARIES, Nainital, India) and the Indian Space Research Organization (ISRO, Bangalore, India) is a combined effort to find $\delta$ Sct- and roAp-type pulsations in Am and Ap, stars respectively, and to study them in more detail (Martinez et al. 2001; Joshi et al. 2006, 2009, 2016).

\section{Observations and Analysis}

Target selection is one of the basic criteria in detecting variable stars. There is no firmly established selection criterium for detecting new Ap/Am variables. The criterion we used is based on Strömgren photometric indices. The candidate Ap/Am stars are those having Strömgren indices similar to known roAp stars (Joshi et al. 2016) chosen from various database (Renson et al. 1991, Hauck \& Mermilliod 1998, Houk \& Swift 1999, Bychkov et al. 2003).

High-precision photometric observations of candidate CP stars were carried out using the ARIES high-speed photoelectric photometer (Ashoka et al. 2001) attached to the 1.04-m Sampurnanand telescope at ARIES and the Modular Photometer attached to the 0.5-m telescope at SAAO Sutherland. Each candidate star was observed continuously with $10 \mathrm{sec}$ integrations through a Johnson $B$ filter with an aperture of $30^{\prime \prime}$. Each star was initially monitored for a period of 1 to 3 hours with occasional interruptions to measure the sky background. To maximize the chances to detect variability, each candidate was observed several times.

The resulting time series of photometric data were visually inspected to identify and remove the bad data points. They were corrected for coincident counting losses and the interpolated sky background was subtracted. After that, the correction for the mean atmospheric extinction of the site was applied. The time of mid-point of each individual data point was then converted into heliocentric Julian dates (HJD) with an accuracy of $10^{-5}$ day $(\sim 1 \mathrm{sec})$. The final reduced data is a time-series of HJD and $\Delta B$ magnitude with respect to the mean of the light curve. The Fourier transform of the cleaned light curves were calculated to search for the periodic signals. The amplitudes corresponding to long term variations due to sky transparency were removed from the Fourier transform. This is also known as a prewhitening procedure.

\section{Results}

\subsection{Positive results}

Under the Nainital-Cape survey, we discovered rapid pulsation in an Ap star HD12098 and $\delta$ Sct type pulsations in seven Am stars. The basic parameters of these new variables are given briefly here.

1. HD 12098: This is the first roAp star discovered under the Nainital-Cape survey. The period of this star is 7.6 min, which shows amplitude modulation with a maximum amplitude of 3-mmag (Girish et al. 2001).

2. HD 13038: This object is classified as an A3-type star. It pulsates with periods of $28.7 \mathrm{~min}$ and $36.23 \mathrm{~min}$. The beating of these two frequencies is seen in the light curve. The Ap or Am nature of this star is unclear (Martinez et al. 2001).

3. HD 13079: It is shown to be pulsating with a $78 \mathrm{~min}$ period and a peak-to-peak B amplitude of $0.02 \mathrm{mag}$. This is a fundamental-mode pulsator near the zero-age main-sequence (ZAMS) (Martinez et al. 2001).

4. HD 25515: The star pulsates with a period of $2.8 \mathrm{~h}$. A clear amplitude modulation is observed in the light curve of this star (Joshi et al. 2009). 
5. HD 98851: This is an Am star of spectral type F2. The pulsation periods of this star are 80.4 min and $162 \mathrm{~min}$. Alternating high and low amplitude cycles in the light curves indicate a nearly sub-harmonic period ratio of 2:1 (Joshi et al. 2003).

6. HD 102480: This is an Am star of spectral type F1. This star pulsates with periods of $156.0 \mathrm{~min}$ and 84 min with alternating high and low amplitude variations with period ratio close to 2:1 (Joshi et al. 2003).

7. HD 113878: This is an Am star pulsating with a period of $2.31 \mathrm{hr}$, typical for $\delta$ Sct stars. The spectral type of this star is F1/F3 indicating that HD 113878 is a marginal Am star (Joshi et al. 2006).

8. HD 118660: This is a metallic and evolved peculiar stars of spectral type A8V. It is a multiperiodic $\delta$ Sct variable pulsating with a principal period of about $1 \mathrm{hr}$. A secondary period of $2.52 \mathrm{hr}$ is also apparent (Joshi et al. 2006).

\subsection{Null results}

The null refers to stars with a non-detection of light variation during our observations. Null results are important both for the observational characterization of the observing site and for making a distinction between pulsating and non-pulsating Ap/Am stars. However, one should be careful to classify an object as a null result knowing that Ap/Am variables show amplitude modulation. For example, based on the extensive photometric and spectroscopic observations of the Ap star HD 103498 (Joshi et al. 2010) and the Am star HD 207561 (Joshi et al. 2012), the periodic variability observed in these stars could not be confirmed with new data. Therefore, the null results are worth to be retested for variability in near future. A total of 345 candidates were tested for variability under the Nainital-Cape survey, out of which 337 turned out to be candidate null results (Joshi et al. 2016).

We have compiled various astrophysical parameters of the objects observed under Nainital-Cape survey and of known roAp stars using standard relations and data taken from the literature (Joshi et al. 2016). This compilation is a very important tool that serves as a basis for the future study of CP stars. The derived luminosities and temperatures can be used to compare the positions of null results, $\delta$ Sct variables and roAp stars in the Hertzsprung-Russell diagram (Joshi et al. 2016). This will help to distinguish between pulsating and non-pulsating $\mathrm{CP}$ stars.

\subsection{Noise Characterization}

The atmospheric noise at an observing site affects the detection of photometric variability. For the bright stars ( $\sim 10 \mathrm{mag})$, atmospheric noise is dominated by scintillation noise over the photon noise. Using an updated version of the Young $(1967,1974)$ approximation by Osborn et al. (2015), the theoretical values of scintillation noise for the Sutherland and Nainital sites were estimated (Joshi et al. 2016). Our analysis shows that scintillation noise for the ARIES site for a telescope with a diameter of $1.04-\mathrm{m}$ is $0.0338 \mathrm{mmag}$. Similarly, for the SAAO site, the estimated noise for a telescope with a diameter of $0.5-\mathrm{m}$ is $0.0433 \mathrm{mmag}$. When scaled to the diameter of the telescope used at ARIES (1.04-m), it becomes 0.0340 mmag. For the moderately larger 3.6-m Devasthal Optical Telescope (DOT), resecently installed at the ARIES site, the scintillation noise is $0.0217 \mathrm{mmag}$. Therefore, the new observing facilities at Devasthal would be very useful for the asteroseismic studies of Ap and Am stars.

\section{Conclusions}

The Nainital-Cape survey is an ongoing survey program to search and study CP variable stars. Under this programme, a total of 345 candidate $\mathrm{CP}$ stars has been observed till date. A new roAp 
star HD12098 and seven $\delta$ Sct-type pulsating stars (HD 113878, HD 98851, HD 13079, HD 102480, HD 25515, HD 13038 and HD 118660) were discovered. The basic parameters of these stars were also determined by spectroscopic modeling by Joshi et al. (2017). Our photometric analysis suggests that a detection level of about $0.6 \mathrm{mmag}$ in the frequency range $1-5 \mathrm{mHz}$ is achieved in this survey program. With the development of new observational facilities at Devasthal, ARIES Nainital, the study of CP stars is expected to achieve new heights in the area of asteroseismology.

\section{Acknowledgements}

We are thankful to Prof. Don Kurtz (UCLAN, UK), Prof. Peter Martinez (UCT, South Africa), Prof. Ram Sagar (IIA, Bengaluru, India), Prof. S. Seetha, Drs. V. Girish and B. N. Ashoka (ISRO, Bengaluru, India) for their never ending support. NKC would like to thank the Director of ARIES (Nainital, India) for the local hospitality and facilities provided during his visit to ARIES. This research has made use of the SIMBAD database, operated at CDS, Strasbourg, France, NASA's Astrophysics Data System and ESA's Hipparcos database.

\section{References}

Ashoka B. N., Kumar, B. V. C. et al. 2001, JApA, 22, 131

Bychkov V. D., Bychkova L. V., Madej J. 2003, 407, 631

Catanzaro G., Ripepi V. 2014, MNRAS, 441, 1669

Girish V., Seetha S., Martinez P. et al. 2001, A\&A, 380, 142

Hou W., Luo A., Yang H. et al. 2015, MNRAS, 449, 1401

Hauck B., Mermilliod M. 1998, A\&AS, 129, 431

Houk N., Swift C. 1999, Michigan catalogue of two-dimensional spectral types for the HD Stars, 5

Joshi S., Joshi Y. C. 2015, JA\&A, 36, 33

Joshi S., Girish V., Sagar R. et al. 2003, MNRAS, 344, 431

Joshi S., Mary D. L., Martinez P. et al. 2006, A\&A, 455, 303

Joshi S., Mary D. L., Chakradhari N. K., Tiwari S. K., Billaud C. 2009, A\&A, 507, 1763

Joshi S., Ryabchikova T., Kochukhov O. et al. 2010, MNRAS, 401, 1299

Joshi S., Semenko E., Martinez P. et al. 2012, MNRAS, 424, 2002

Joshi S., Martinez P., Chowdhury S. et al. 2016, A\&A 590, A116

Joshi S., Semenko E., Moiseeva A. et al. 2017, MNRAS, 467, 633

Kurtz D. W. 1982, MNRAS, 200, 807

Kurtz D. W., Martinez P. 2000, Balt. Astronomy, 9, 253

Martinez P., Kurtz D. W., Ashoka B. N. et al. 2001, A\&A, 371, 1048

Osborn J., Föhring D., Dhillon V. S., Wilson R. W. 2015, MNRAS, 452, 1707

Preston G. W. 1974, ARA\&A, 12, 257

Renson P., Gerbaldi M., Catalano, F. A. 1991, A\&AS, 89, 429

Smalley B., Kurtz D. W., Smith A. M. S. et al. 2011, A\&A, 535, A3

Wolff S. C. 1983, The A type stars: Problems and perspectives, NASA SP-463

Young A. T. 1967, AJ, 72, 747

Young A. T. 1974, ApJ, 189, 587 